\title{
Quasi-annual variations in evolution of large-scale solar magnetic field
}

\author{
R.N. Ikhsanov and V.G. Ivanov \\ Central Astronomical Observatory at Pulkovo, Saint-Petersburg, Russia; E-mail: \\ solar1@gao.spb.ru
}

\begin{abstract}
We study variations of the solar large-scale magnetic field (LMF) with period $\approx 1.0-1.3$ years by observational data of Stanford (1976-2004) and Kitt-Peak (1970-1984).
\end{abstract}

Cyclic evolution of LMF can be divided, in accordance with type of its activity, into two phase (Ikhsanov \& Ivanov 2001). On phase I, which includes $\pm 2-3$ years near the 11-year cycle maximum, notable changes of the magnetic filed neutral line take place, including the polar reversal of the solar magnetic field. In the rest of the cycle (phase II) the magnetic field of constant polarity is observed in each hemispheres, and oscillations of the neutral line relative to the equator do not exceed the bounds of latitude $\pm\left(0^{\circ}-30^{\circ}\right)$. Latitude-time evolution of LMF on phase I for different longitude zones was studied in our previous papers (Ikhsanov \& Ivanov 2002, Ikhsanov \& Ivanov 2003). Now we are interested in longitude and latitude periodical perturbations in evolution of the LMF. Calculating of distances between successive hills of isogausses on the north and south hemispheres, one can find their recurrence with period $1.22 \pm 0.13$ years. One can see that successive maxima of latitude deviation of the neutral line shifted in time for the same interval $\approx 1.3$ years.

One can see this regularity in Fig. 1, which presents succession of latitude-longitude diagrams diagrams, with time averaging for every diagram and time shift between adjacent ones being $1 / 3$ year. In cycle 21 groups of several adjacent diagrams are presented, in which maxima of latitude deviation of the neutral line from the equator practically do not change their longitudinal positions. However, in adjacent groups (marked by arrows) these maxima shift rather essentially. The groups selected in Fig. 1 consist of three or four successive diagrams, i.e. in 7.33 years 6 successive longitudinal shifts were observed, with typical time between their shifts being on average 1.22 years and longitudinal dimension of the shifts varies from $90^{\circ}$ to $180^{\circ}$. The similar picture can be seen in cycle 22 . On average for the selected intervals in two cycles oscillation of the neutral line configuration with typical period $1.23 \pm 0.16$ years are observed.

Previously many authors found the period $\approx 1.3$ years by statistical methods in different indices of solar activity (see, e.g, Ikhsanov \& Ivanov 2003). In paper of Ikhsanov \& Miletsky (1996) it was found, with use of monthly averaged sunspot areas, that process of quasi-annual oscillations in 11-year cycle take place continuously, with some variations which depend upon power of the annual peaks. However, in this case we can directly observe how the oscillations of the LMF take place. Positions of latitude variations of maxima of the LMF neutral line are related with their longitude shift with period 1.01.3 years $(1.23 \pm 0.16$ year). This periodicity are close to the period $\sim 1.3$ years, which was found by helioseismological methods in variations of the solar rotation in the region of tachocline (Howe et al. 2002). Therefore, emerging of the LMF from inner layers of the Sun are organized in the form of discrete packets with duration from 1.0 to 1.3 years (13-17 Carrington rotations). 


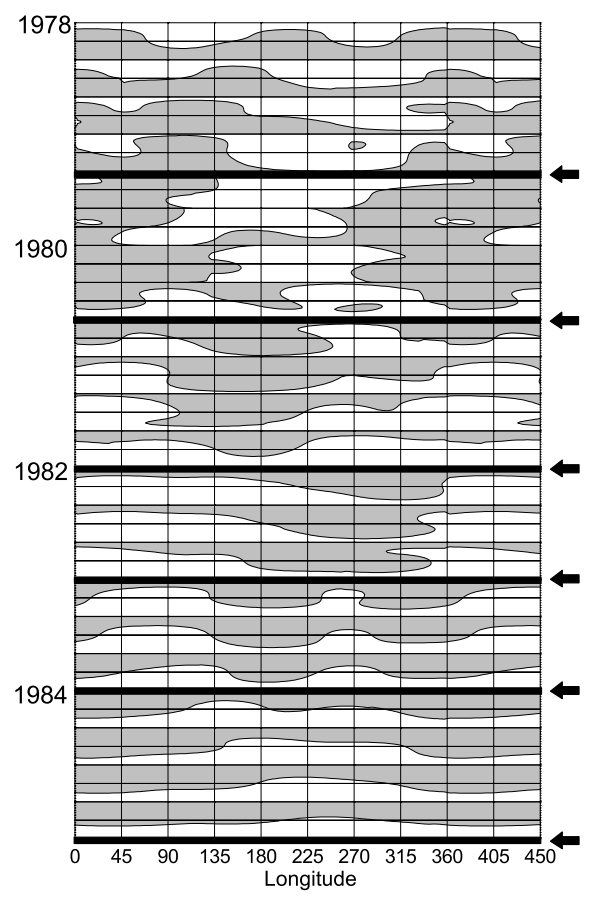

Figure 1. Evolution of the neutral line on successive (arranged from top to bottom) latitude-longitude diagrams

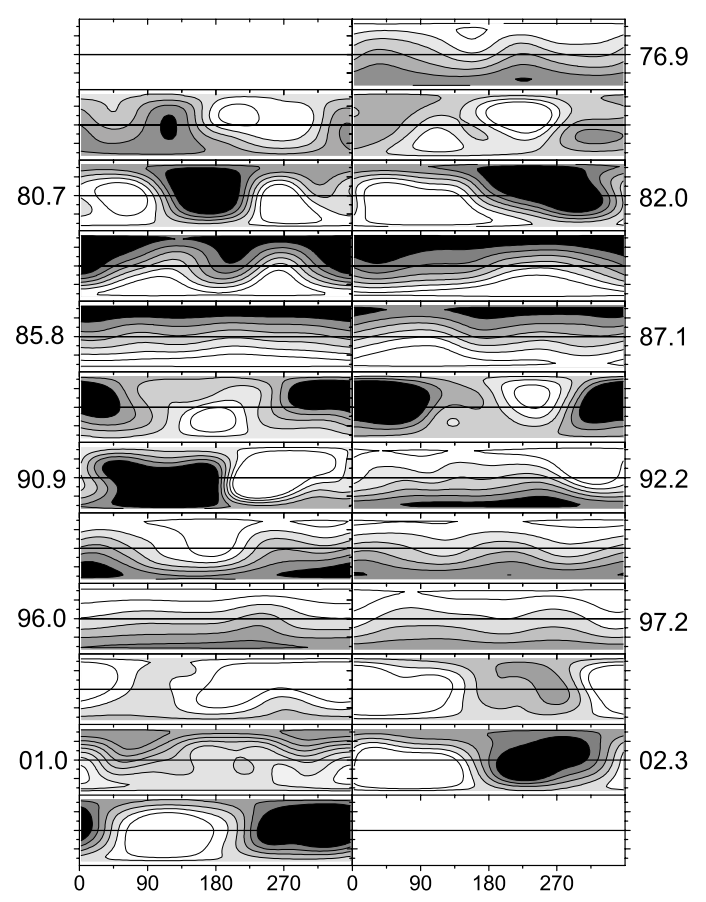

Figure 2. Synoptic charts of the LMF by data of Stanford

In Fig. 2 synoptic charts of the LMF by data of Stanford, from 1970 to 2004 year with 17 -rotation averaging are presented. The zero point of the time scale is chosen, according to Fig. 1, in the moment 1982.0.

We can conclude that:

- In epoches of the solar magnetic field polar reversal a typical picture with the LMF equatorial dipole is observed on the synoptic charts with beginning in 1979.5, 1989.6, 1999.8.

- The synoptic charts that mark ends of the LMF phase I and begin in moments 1982.0, 1990.9, 2002.3 correspondingly are especially characteristic. On these charts the LMF of one sign occupies mainly areas in ranges of longitudes $0^{\circ}-180^{\circ}$ or $180^{\circ}-360^{\circ}$.

- In patterns of the LMF distribution for different cycles there are both some similarities and essential differences. For instance, in the above mentioned synoptic maps in cycles 21-22 and 22-23, as distinct from the adjacent cycles 20-21, longitudinal positions of the magnetic field polarity switch their places. Such difference is also observed between pairs of even $(20,22)$, on the one hand, and odd $(21,23)$, on the other.

\section{References}

Howe, R., Christensen-Dalsgaard, J., Hill, F. et al. 2002 Science 287, 2456.

Ikhsanov, R.N. \& Ivanov, V.G. 2001 In Proceedings of the conference "The Sun in epoch of the polar reversal", Saint-Petersburg, 28 May - 1 June 2001, p.181.

Ikhsanov, R.N. \& Ivanov, V.G. 2002 Izvestija GAO 216, 531.

Ikhsanov, R.N. \& Ivanov, V.G. 2003 In Proceedings of the conference "Climatic and ecological aspects of solar activity", Saint-Petersburg, 7-11 June 2003, p.189.

Ikhsanov, R.N. \& Miletsky, E. V. 1996 Izvestija GAO 211, 167. 